\title{
Molecular Dynamics Simulations of Signal Sequences at a Membrane/Water Interface
}

\author{
Elizabeth P. G. Arêas,,$^{*}{ }^{\dagger}$ Pedro G. Pascutti, ${ }^{\ddagger}$ Shirley Schreier,${ }^{\S}$ Kleber C. Mundim, ${ }^{\perp}$ and \\ Paulo M. Bisch ${ }^{\ddagger}$ \\ Departamento de Química Fundamental, Instituto de Química, Universidade de São Paulo, CP 26077, CEP \\ 05599-970 São Paulo, SP, Brazil; Instituto de Biofisica, Universidade Federal do Rio de Janeiro, Rio de \\ Janeiro, RJ, Brazil; Departamento de Bioquímica, Universidade de São Paulo, SP, Brazil; and Instituto de \\ Fisica, Universidade Federal da Bahia, Salvador, BA, Brazil
}

Received: April 11, 1995; In Final Form: July 7, $1995^{\otimes}$

\begin{abstract}
A recently developed software has been used to model peptides at a cytoplasm/membrane mimetic environment where the interface is represented by a discontinuity in the dielectric constant. Molecular dynamics and energy minimization procedures available in the program were applied to a wild type and to a $50 \%$ active mutant $\left(\Delta 78 \mathrm{r}_{1}\right)$ peptide signal sequence of a $\lambda E$. coli receptor (maltoporin). Modeling has been performed for both random coiled and constrained helical structures. As a general feature, the presence of the dielectric discontinuity induced the movement of the molecules' center of mass toward the interface. A decrease in the energy along interface crossing (from $\epsilon=80$ to $\epsilon=2$ ) was observed and interpreted as an indication of their affinity for the lipid-mimetic phase. Distinct patterns of migration were recognized for each sequence, as well as in different simulated conditions for a same peptide. The random coiled peptides easily cross the interface, showing a tendency to go into the nonpolar phase, whereas constrained helical sequences tend to stay at the interface. Potential barriers and potential wells were identified in the modeling space for constrained helical peptides, which have been shown to be dependent on the peptide primary sequence, on the conformational restrictions imposed, and on the charge state of the peptide terminals.
\end{abstract}

\section{Introduction}

The application of molecular dynamics methods has proved to be of particular importance in the development of structural studies of biomolecules in the recent past decades, both as a complementary tool to methods such as X-ray crystallography and nuclear magnetic resonance spectroscopy (NMR) and as an instrument for structural studies of proteins by homology to related molecules of known structure. ${ }^{1-5}$ Its use in the modeling of biological molecules has represented great progress in the design of molecular structures with desired specific properties. ${ }^{6}$ Such products can be obtained synthetically or by genetic engineering procedures, as in the case of proteins. Important advances toward a better understanding of the protein folding problem can be found in many recent contributions, such as in those compiled by Merz, Jr., and Le Grand. ?

The use of molecular dynamics procedures for modeling purposes can lead ultimately to the understanding of the dynamic situations compatible with experimental observations, permitting a formal definition of the conditions that would be theoretically expected to produce the observed behavior. ${ }^{2,8}$ That could, in principle, allow the inference of patterns of behavior and structure for other systems and situations of interest which lack experimental information. In the study of molecular systems where some structural information is available, molecular dynamics has proved to be of great importance, opening a new road of investigation where fundamental considerations can be checked against experimental evidence. ${ }^{4,9}$

We have undertaken here the study of two homologous but functionally distinct signal sequences, about which NMR

\footnotetext{
* To whom correspondence should be addressed.

† Departamento de Química Fundamental, Universidade de São Paulo.

* Universidade Federal do Rio de Janeiro.

Departamento de Bioquímica, Universidade de São Paulo.

$\perp$ Universidade Federal da Bahia.

- Abstract published in Advance ACS Abstracts, September 1, 1995.
}

spectroscopic information is available. ${ }^{10,11}$ Signal sequences are peptide fragments encountered at the $\mathrm{N}$ terminal end of proteins, which are involved in cell signaling processes. ${ }^{10,12}$ An important aspect related to peptides is the great number of degrees of freedom for linear conformations. This can lead to several conformations with equivalent values of minimum energy. Membrane-binding peptides, however, will have their degrees of freedom diminished when placed at the water/membrane interface, due to the different affinity of amino acid residues for the aqueous medium and for the nonpolar environment. Modeling should allow preferential conformations to be detected in this kind of environment, making possible a better understanding of their biological role at the molecular level.

Signal sequences are relatively short peptide sequences (1530 amino acids) responsible for critical functions related to protein targeting and export. ${ }^{11,13}$ Their most striking common conformational feature is the internal helical core, whose stability is related to the biological signaling function. The affinity of signal sequences for lipids has been well established, as also has their capacity to form helices in membrane-mimetic environments, such as in certain organic solvent media. ${ }^{10,11} \mathrm{An}$ expressive amount of spectroscopic work has been performed on these systems, such as circular dichroism (CD), infrared (IR), and NMR, ${ }^{10-12}$ and they indicate that functional sequences show a strong preference for $\alpha$-helical secondary structure.

Due to their small size and their role in crucial cell functions, signal sequences can be used as models for the characterization of secondary structure formation in isolated fragments of polypeptide chains, in attempts to understand the relationship between primary sequence, folding, and function. We have studied here the wild type (WT) and a mutant signal sequence $\left(\Delta 78 \mathrm{r}_{1}\right)$ of the lamB gene product, a protein of the external membrane of $E$. coli, also known as $\lambda$ receptor or maltoporin. The wild type peptide presents a 25-residue sequence (MMITLRKLPLAVAVAAGVMSAQAMA). Gierash and collaborators $^{11}$ have produced mutants that presented absent or partial 
degrees of functionality. A mutant $(\Delta 78)$ lacking four sequential residues (LAVA) in the hydrophobic core (from residue 10 to residue 13) had its capacity for helix formation completely abolished and presented no biological functionality. However, $50 \%$ activity was reestablished by replacing a Gly residue in the $\Delta 78$ mutant (G13) with a Cys residue. The new, partially active mutant was called $\Delta 78 r_{1}$. Its amino acid sequence is therefore MMITLRKLPVAACVMSAQAMA. The alignment and numbering of the peptide sequences are depicted below:

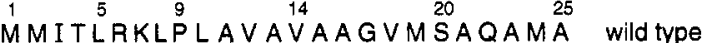

$$
\begin{aligned}
& \text { MMITLRKLP-- - VAACVMSAQAMA } \triangle 78 r_{1} \text { mutant }
\end{aligned}
$$

\section{The Program}

A program has been developed for the modeling of molecules of biological interest in aqueous and nonpolar media as well as at the interface between them. ${ }^{1}$ The software establishes two effective media with different continuous dielectric constants, separated by a surface. The dielectric discontinuity is taken as an approximation of a cytoplasm/membrane environment. This program, named "THOR", has been developed by some of the authors ${ }^{1}$ with the aim of allowing molecular modeling of biomolecules at simulated biological interfaces.

The software accepts Cartesian or internal atomic coordinates as input data. The molecule's chemical linkages should be also specified, and a topology table should be built, containing specific parameters associated with the molecule. The parameters used, which characterize the force field, are those from GROMOS $^{14}$ for protein data bank.

An energy minimization procedure is available. It optimizes the molecular geometry making use of the "steepest descent" algorithm. Molecular dynamics uses the Verlet algorithm. 15,16 Parameters monitored during the dynamics include temperature, kinetic and potential energy, dihedral angles, and $x$-coordinate for the center of mass. Sets of internal as well as Cartesian coordinates are produced for the structures generated during the dynamics.

The Force Field. The force field for the system to be modeled is expressed in the program by a superposition of simple terms for a system of $N$ bodies. Only polar hydrogen atoms (bound to oxygen or nitrogen) are explicitly taken into account, while $\mathrm{CH}_{2}$ and $\mathrm{CH}_{3}$ groups are taken as united atoms (pseudoatoms).

The total potential energy for a given molecule is expressed in the form

$$
\begin{aligned}
& V\left\{\left(\vec{r}_{i}\right)\right\}=V\left(\vec{r}_{1}, \vec{r}_{2}, \ldots, \vec{r}_{N_{\mathrm{ar}}}\right)=1 / 2 \sum_{\text {bonds }} K_{b}\left[b-b_{0}\right]^{2}+ \\
& 1 / 2 \sum_{\text {angles }} K_{\theta}\left[\theta-\theta_{0}\right]^{2}+1 / 2 \sum_{\text {impropers }} K_{\zeta}\left[n-\zeta_{0}\right]^{2}+\sum_{\text {dihedrals }} K_{\varphi} \\
& \times[1+\cos (n \varphi-\delta)]+\sum_{\text {nonbonded pairs }}\left[\frac{C_{12}}{r_{i j}{ }^{12}}-\frac{C_{6}}{r_{i j}{ }^{6}}+\frac{q_{i} q_{j}}{4 \pi \epsilon_{0} \epsilon_{r} r_{i j}}\right]
\end{aligned}
$$

where the first four terms to the right of the equation represent short-range interactions, namely, harmonic potentials related to the covalent bond between pairs of atoms, the $\theta$ angle between every three bound atoms, the improper dihedral angle for special groups of four atoms where a nearly fixed position must be maintained, and the senoidal dihedral torsion. The last two terms represent the long-range interactions, van der Waals and Coulomb, respectively. First and second neighbors are excluded from these last two terms.

The Interface. The membrane/water interface is expressed by a discontinuity in the dielectric constant, taking into account the different electrical polarizability of the aqueous and the hydrocarbon phases. Then, the electrostatic interaction between nonbounded atoms at this interface will be renormalized by the influence of the dielectric discontinuity. ${ }^{1}$ The polarization field produced at the surface of discontinuity by a point charge can be calculated by the method of images. ${ }^{17}$ A fictitious charge is placed in the opposite phase; the distance and the value of the image charge are fixed by taking the appropriate electrical boundary conditions at the surface.

When two charges $i$ and $j$ are on the same side of the interface, the potential on $i$ due to $j$ will have a contribution due to the image of $j$ (i.e., $j^{\prime}$ ), so that the Coulomb potential on $i$ will be expressed by

$$
V_{i}=\frac{1}{4 \pi \epsilon_{1}} q_{j}\left\{\frac{1}{r_{i j}}+\frac{\epsilon_{1}-\epsilon_{2}}{\epsilon_{1}+\epsilon_{2}} \frac{1}{r_{i j}}\right\}
$$

where

$$
r_{i j}=\left[\left(x_{i}-x_{j}\right)^{2}+\left(y_{i}-y_{j}\right)^{2}+\left(z_{i}-z_{j}\right)^{2}\right]^{1 / 2}
$$

is the distance between $i$ and $j$ and

$$
r_{i j^{\prime}}=\left[\left(x_{i}+x_{j}-2 x_{S}\right)^{2}+\left(y_{i}-y_{j}\right)^{2}+\left(z_{i}-z_{j}\right)^{2}\right]^{1 / 2}
$$

is the distance between $i$ and $j^{\prime}$, and $x_{\mathrm{S}}$ is the Cartesian coordinate of the surface between the two media. When two charges are at different sides of the interface, the method of images gives the following result:

$$
V_{i}=\frac{1}{4 \pi} \frac{q_{j}}{\left(\epsilon_{1}+\epsilon_{2}\right) / 2} \frac{1}{r_{i j}}
$$

We note that the above terms account for the effect of the interface on the pair interaction terms only, the self-induced field created by a single charge being ignored here. The latter will be a pure solvent effect which should be corrected by other solvent effects not treated here. ${ }^{18}$ Since we are most interested in the molecular conformational changes at the interface, we prefer only to consider the renormalization of the pair interactions, leaving solvent effects for further investigations.

\section{Modeling Strategy}

We have made use of two differently terminal charged peptides: a zwitterionic and a neutral terminal peptide form. The zwitterionic form has been used, in accordance with the expected ionization for the $\mathrm{N}$ and $\mathrm{C}$ terminal groups at $\mathrm{pH} 4.0$, so as to allow a better comparison of the modeled data with the conditions in which the experimental NMR data were obtained. ${ }^{10}$ The neutral form has been studied more specifically as a means of assessing the effect of the terminal charges on the helix stability. The ionization state of ionizable groups in Arg and Lys have been taken into account in both cases.

We have applied distinct modeling strategies so as to approach different aspects in the context of this work:

(a) Starting from an initial random coil polypeptide conformation in a polar medium $(\epsilon=80)$, the migration through an $\epsilon=80 / \epsilon=2$ interface has been followed for the two peptides. An optimization of the molecular geometry in $\epsilon=80$ was performed prior to molecular dynamics. The latter was applied initially in this polar medium, for a period of time sufficient to achieve thermal equilibrium at $300 \mathrm{~K}$, in our case not less than 50 ps. Steps of 0.5 fs were employed in all dynamics. Atomic coordinates were produced at regular intervals so as to allow the visualization of the structures generated along the 


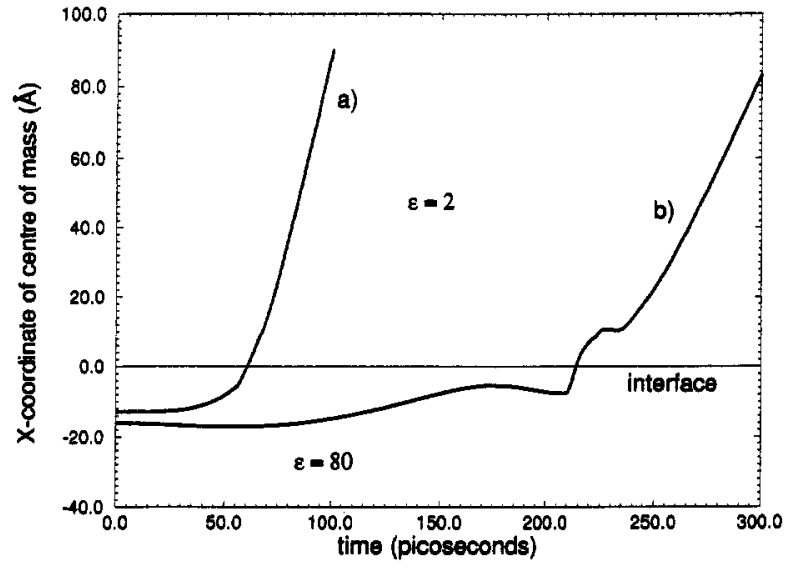

Figure 1. Random coiled peptide migration across the interface: (a) $\Delta 78 r_{1}$ mutant; (b) wild type.

dynamics. Potential, kinetic, and total energy changes were recorded along the simulation. An interface to a nonpolar medium $(\epsilon=2)$ was introduced following thermalization in water.

(b) Starting from an imposed partial helical conformation in a continuous medium (both $\epsilon=80$ and $\epsilon=2$ media were studied), conditions were investigated concerning the helix stability and its dependence on the molecule's total geometry and on terminal charges. The imposed helical conformation conforms to NMR evidence ${ }^{10}$ for the peptides at $2 \mathrm{mM}$ concentration in a 50/50 (by volume) TFE (trifluorethanol) $/ \mathrm{H}_{2} \mathrm{O}$ mixture, at $\mathrm{pH} 4.0$. In accordance with this, a helix was introduced from residue Leu8 to Met24 in the wild type peptide and from residue Leu 8 to Ala23 in the mutant molecule. The helical conformation was imposed through the choice of appropriate dihedral backbone, so as to conform to the values expected for an $\alpha$-helix. High force constant values were applied to the dihedral angles involved in the helix, as a means to favor the persistence of the helical structure during some stages of the modeling. Such imposed helical conformations are called in this paper "constrained helices". The modeling in the continuous nonpolar medium $(\epsilon=2,50 \mathrm{ps})$ was followed by the introduction of an interface to a polar environment $(\epsilon=$ 80). When modeling started in a polar medium, an interface to a nonpolar medium was introduced in a similar way. For the wild type peptide in the low dielectric side, the helical constraints were abolished (by returning the force constants to their normal values) after 100 and 200 ps for the neutral and zwitterionic peptides, respectively, in order to investigate their stability. A study of the potential profile across the $x$-coordinate of the modeling space has been also performed by taking sequential optimization steps for the constrained helical and random coiled peptides as a function of their distance from the discontinuity surface.

\section{Results and Discussion}

Modeling from an Initial Random Coil Conformation. As a general feature, the peptides evolved during the dynamics in water (a continuous medium of $\epsilon=80$ ) from an initially rather stretched, random coil conformation to increasingly folded globular structures. That had already been observed for the mutant peptide and indicated in a previous communication. ${ }^{19}$ A displacement of the peptide's center of mass toward increasing $x$ values was induced by introduction of the interface (Figure 1). The mutant peptide showed a much faster interface crossing than the wild type one. In the latter, ca. 200 ps dynamics were needed until a migration rate comparatively as fast into the

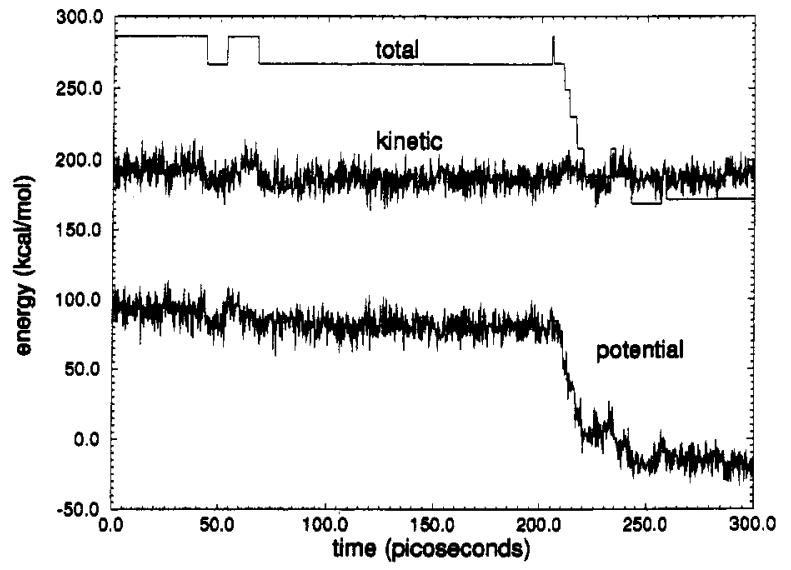

Figure 2. Kinetic, potential, and total energy for random coiled wild type peptide.

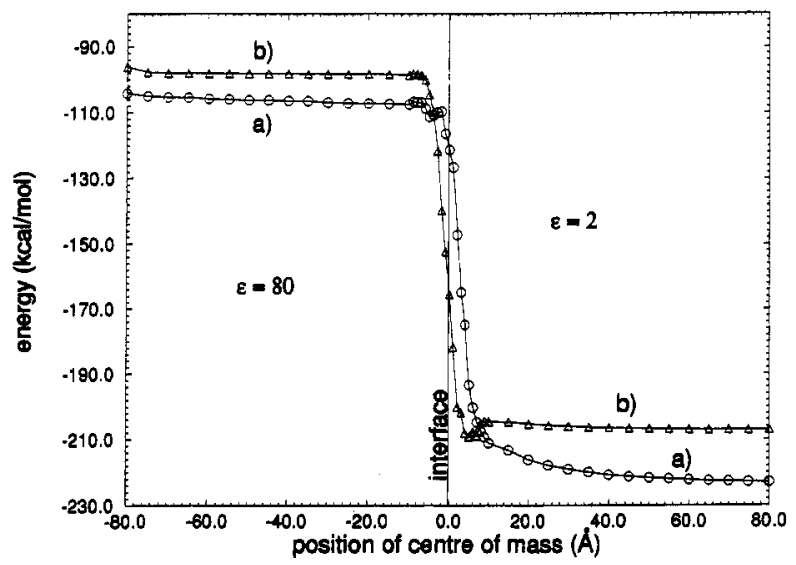

Figure 3. (a) Potential profile for random coiled $\Delta 78 r_{1}$ mutant peptide. Interface at $x=0$. (b) Potential profile for random coiled wild type peptide. Interface at $x=0$.

nonpolar phase was achieved (Figure 1). The pattern of migration is very distinctive, with a steadily forward migration in the case of the mutant sequence and a rather slow entry of the wild type into the nonpolar medium, across the interface (Figure 1). The entry of both peptides into the lipid-mimetic environment caused a decrease in the total and potential energy, indicating their affinity for the membrane. The energy profile presented by the wild type peptide is shown in Figure 2.

A series of optimization procedures (without any dynamics) were applied to the random coiled mutant (Figure 3a) and wild type (Figure $3 \mathrm{~b}$ ) peptides, at different $x$-coordinate values, in order to have a potential profile as a function of distance from the interface (positioned at $x=0$ ). A decrease in the energy values is observed at the proximity of the interface for both cases. However, no significantly deep potential wells were found in these cases (random coiled peptides), as compared to what is observed for the helical sequences (compare with Figure $5 \mathrm{a}, \mathrm{b})$. That made the peptide stabilization at the interface an unlikely event for the random coiled peptides, as can be seen in Figure 1, in spite of the initial apparent stabilization tendency shown by the wild type molecule in the first 200 ps. The occurrence of a small potential well for the wild type sequence (Figure $3 \mathrm{~b}$ ) accounts for that slower interface crossing and apparent stabilization presented by this peptide at smaller dynamics times, as compared to the mutant (Figure 1). However, after a sufficiently long dynamics at $300 \mathrm{~K}$ (ca. 200 ps), the energy barrier at Figure $3 b$ is eventually overcome by the molecular kinetic energy, and the wild type peptide introduces itself into the nonpolar phase at a rate comparable to that of the mutant (Figure 1). The random coiled peptides 


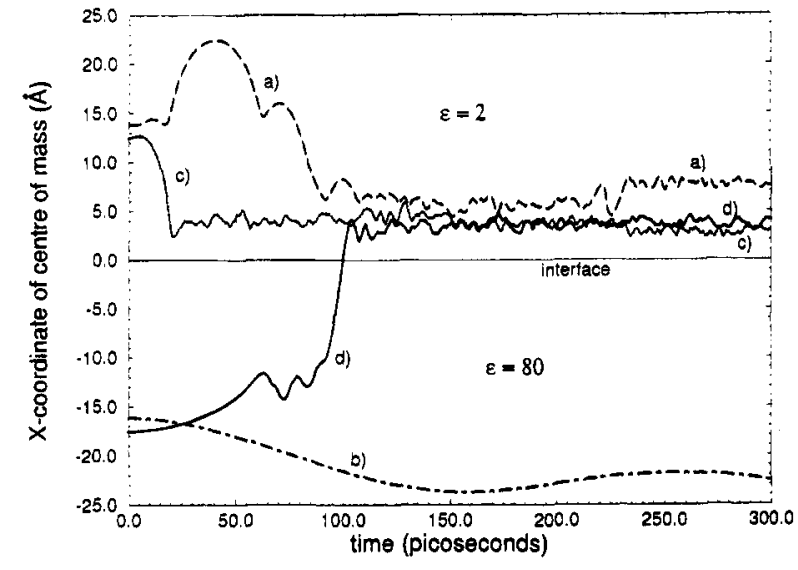

Figure 4. Movement of center of mass for the wild type peptide with constrained helix: (a) peptide in the zwitterionic form; dynamics starting at $\epsilon=2$; (b) peptide in the zwitterionic form; dynamics starting at $\epsilon=$ 80; (c) peptide in the terminal neutral form; dynamics starting at $\epsilon=$ 2; (d) peptide in the terminal neutral form; dynamics starting at $\epsilon=$ 80 .

have therefore shown a more hydrophobic behavior than what has been observed for the helical systems (Figure 4), in terms of a clear preference for the interior of the nonpolar phase.

Modeling from a Partially Helical Initial Conformation. Nonconstrained helical conformations have been initially imposed to specific regions in the peptides to conform to experimental evidence, ${ }^{10}$ as already described. Since no further experimental structural information is provided in the literature regarding a distinction of the kind of helix encountered in those peptides, the $\phi$ and $\psi$ angular values used were those described for the most prevalent $\alpha$ helical conformation.

The maintenance of a nonconstrained helical conformation along the dynamics showed to be critically dependent on factors involving the geometry of the molecule as a whole. At the $\mathrm{N}$ terminal region of the lamB peptides the helix is flanked by a Pro, well-known as a helix-disrupting residue. Its occurrence in the helix in those signal sequences has been pointed out ${ }^{10}$ as one of the factors not favoring helix stability at the N-terminal region of the peptide. Another factor contributing to helix instability is the interaction of the helix dipole with the molecule positively charged end. We have noted a definite tendency for helix disruption at that point in the $\mathrm{N}$ terminal end of the peptide molecule. Some instability of the helix has also been observed during the modeling at the $\mathrm{C}$ terminal region. That coincides with the point where a Gly (Gly17) residue occurs. That instability is expressed by a tendency of the helix to alter more significantly its backbone dihedral angle values from that point toward the $C$ terminal end. Despite its role in affecting the helix stability, we observed that the glycine residue effect is not, however, able to cause a complete unfolding of the nonconstrained helical structure along the dynamics, which is in accordance with NMR observations. ${ }^{10}$

We have observed a dependence of the wild type peptide conformation on the terminal charge status of the nonconstrained helical molecule, when modeled in a continuous medium of $\epsilon$ $=2$. The approximation of the molecule's oppositely charged groups is made particularly intense in a medium with low dielectric constant. For a relatively small polypeptide chain, such an approximation can represent a significant strain to the molecule as a whole, eventually leading to the disruption of the helix.

In order to model the helical peptides migration through the interface, constraints were introduced into the structures to be modeled, in order to conform with experimental evidence. ${ }^{10}$

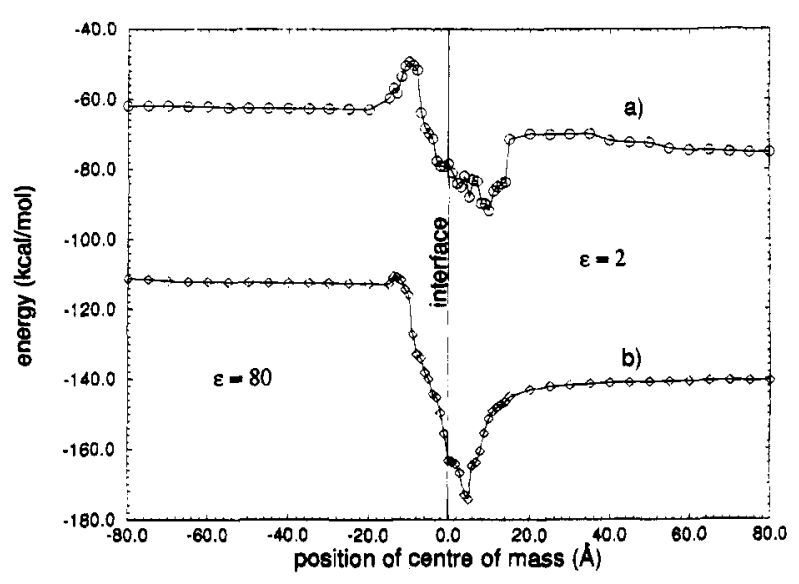

Figure 5. Potential profile for the wild type peptide with constrained helix: (a) in the zwitterionic form; (b) in the terminal neutral form.

According to that, helices were produced for both the wild type and the mutant peptide, by setting the pertinent backbone $\phi$ and $\psi$ dihedral angles to the expected values of an $\alpha$-helix. The conformations were then artificially maintained by application of very high force constant values to those angles, as already mentioned. The mutant helix, however, has shown to be very unstable, and no proper modeling conditions were met that would satisfy its stability. We have therefore restricted our study to the wild type helical peptide.

The movement of the peptide center of mass along the constrained dynamics is shown for the zwitterionic (Figure 4a,b) and neutral terminal peptide (Figure $4 c, d$ ). Zero in the time axis corresponds to the time in which the interface was introduced. Figure 4a shows the migration profile exhibited by the zwitterionic peptide for a dynamics initiated in the nonpolar $(\epsilon=2)$ phase. Figure $4 \mathrm{~b}$ presents the migration observed when the dynamics is applied starting in the polar phase $(\epsilon=80)$. It is possible to see that no convergence is achieved in terms of a final common position for those two situations. Such pattern of behavior can be better understood considering Figure 5a. Here, the potential energy profiles for the zwitterionic peptide is presented. That was done through optimization procedures (no dynamics applied) of the peptide as a function of distance from the interface. That figure shows the presence in the polar phase of an energy barrier $(\Delta E)$ of ca. $14 \mathrm{kcal} / \mathrm{mol}$, with a maximum at around $8 \AA$ from the interface. At the temperature conditions employed $(300 \mathrm{~K})$ in the modeling, such a barrier could not be completely overcome by the kinetic energy of the peptide. A $15 \AA$ wide and $20 \mathrm{kcal} /$ mol deep potential well is, on the other hand, observed on the nonpolar side, centered at around $10 \AA$ from the interface. Such a well would imprison the peptide molecule inside it, ultimately favoring its stabilization there. That conforms to the tendencies shown by the peptides in Figure $4 a$,b, in terms of the final position assumed by the peptide as the dynamics evolves for both situations.

Parts $c$ and $d$ of Figure 4 show the migration pattern presented by the neutral terminal peptide for a dynamics simulation starting at the nonpolar and polar phase, respectively. In that case, the peptide stabilizes at ca. $3 \AA$ from the interface for both situations, which conforms to the energy profile (Figure 5 b) obtained for that form of the molecule by the optimization procedure already described. Because the energy barrier for the neutral terminal peptide at the polar medium (Figure $5 b$ ) is small when compared to that of Figure 5a for the zwitterionic form, the neutral terminal peptide succeeds in overcoming it, causing the trajectories to converge to the well for dynamics starting both in the polar and in the nonpolar phase. The potential well in Figure $5 \mathrm{~b}$ is 


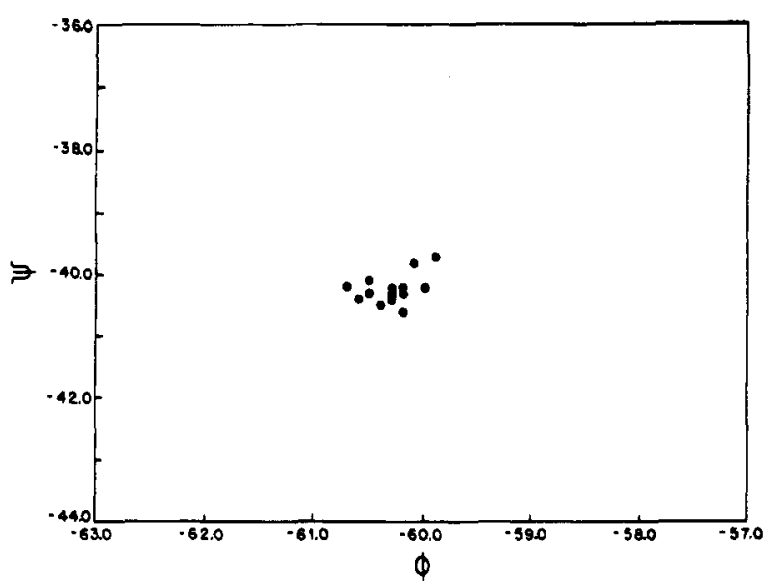

Figure 6. Distribution of $\psi$ and $\phi$ values in mean dynamic conformations for the constrained helical zwitterionic wild type peptide (helical region). Data are averaged values over 50 ps dynamics.

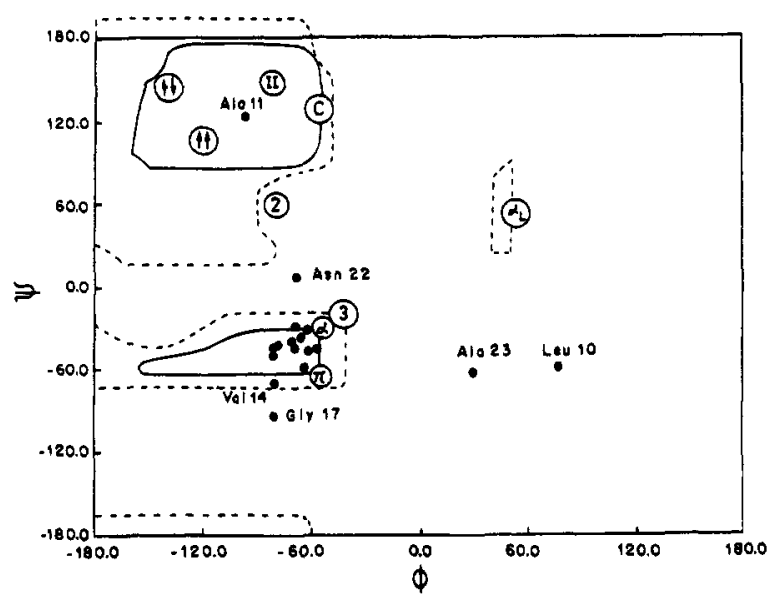

Figure 7. Distribution of $\psi$ and $\phi$ values in mean dynamic conformations for the zwitterionic wild type peptide, after removal of helical constraints. Data refer to averaged values over a $50 \mathrm{ps}$ time interval in the dynamics, from 50 to 100 ps after constraints were removed. A Ramachandran diagram is superimposed, which shows the sterically allowed $\psi$ and $\phi$ angles for poly-L-alanine: regions corresponding to normally allowed $\psi$ and $\phi$ angles are shown in solid lines; those corresponding to "outer limit" van der Waals distances are shown in dashed lines. Conformations are identified as follows: $\alpha$ and $\alpha_{\mathrm{L}}$, righthanded and left-handed $\alpha$-helices; 3 , right-handed 310 helix; $\pi$, righthanded $\pi$ helix; $2,2.27$ ribbon; II, left-handed polyglycine II and polyL-proline II helices; C, collagen helix; $\uparrow \uparrow$ and $\uparrow \downarrow$, parallel and antiparallel $\beta$ pleated sheet, respectively (from: Voet, D.; Voet, J. G. Biochemistry; John Wiley \& Sons: New York, 1990; p 147).

around $30 \mathrm{kcal} / \mathrm{mol}$ deep and a little narrower than the one observed for the zwitterionic molecule in Figure 5a. Although no considerations can be made on the absolute values of the energy minima for both wells, since those are restricted conformations, it is interesting to note that much lower values were shown by the neutral terminal peptide form. A comparison of the energy difference observed between these two minima indicates a higher stability for the neutral terminal peptide as compared to the zwitterionic molecule.

A distribution of mean dynamic conformations (50 ps averaged) for the helical part of peptides is shown in Figures 6-9. The zwitterionic, constrained helical peptide configuration is represented in Figure 6, where the residues in helix are shown. Figure 7 refers to the evolution observed for that structure, after release of the constraints. Figure 8 and 9 present the picture obtained for the neutral peptide form, with averages $(50 \mathrm{ps}$ ) taken 50 and $150 \mathrm{ps}$ after release of the helical constraints, respectively. Ramachandran plots are superimposed onto the plots in Figures 7-9 to permit comparison with allowed regions

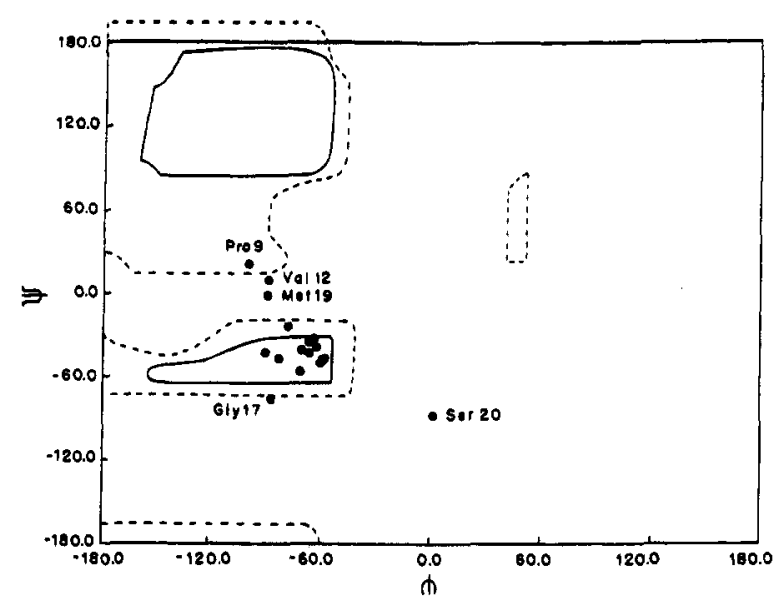

Figure 8. Distribution of $\psi$ and $\phi$ values in mean dynamic conformations for the terminal neutral peptide after removal of helical constraints. Data refer to averaged values over a $50 \mathrm{ps}$ time interval in the dynamics, from 50 to $100 \mathrm{ps}$ after constraints were removed. A Ramachandran plot is superimposed (see details in Figure 7).

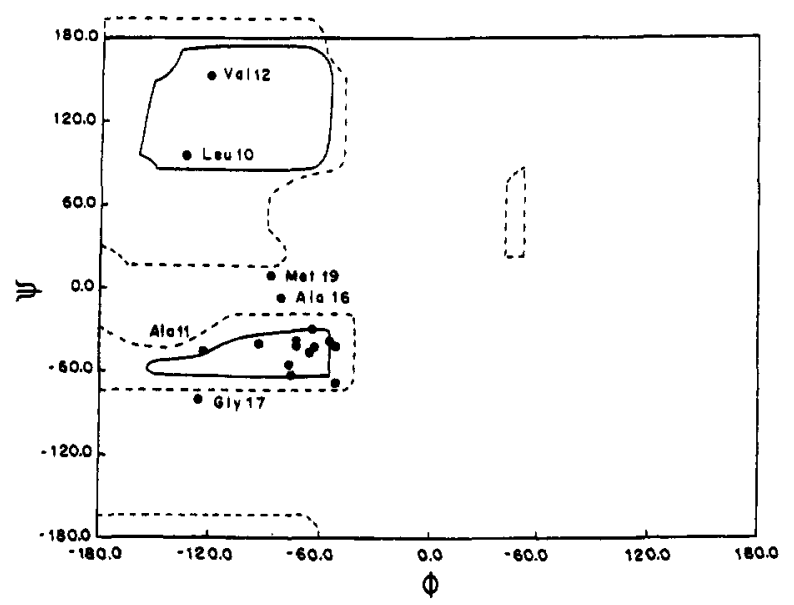

Figure 9. Distribution of $\psi$ and $\phi$ values in mean dynamic conformations for the terminal neutral peptide after removal of helical constraints. Data refer to averaged values over a 50 ps time interval in the dynamics, from 150 to 200 ps after constraints were removed. A Ramachandran plot is superimposed (see details in Figure 7).

for the various secondary structures. It is possible to accompany the drift of some dihedral angles from the expected helical values, with some residues at the $\mathrm{N}$ terminal side of the helical region tending to a $\beta$-sheet conformation. That is the region where proline, a helix disrupting residue, occurs. Gly17 tends consistently to more negative values for both its $\psi$ and $\phi$ dihedral angles (Figures 7-9). Most of the dihedrals, however, remained in the boundaries of the helical region, in some cases for as long as $200 \mathrm{ps}$ after restrictions were removed, as is shown by the neutral peptide form. A comparatively longer persistence of helical conformation was observed for the neutral peptide, possibly due to the nonexistence of the effect of attraction between the $C$ and $N$ terminal ends, $C$ terminal and Arg6, and $\mathrm{C}$ terminal and Lys 7 .

Table 1 shows the average distances between charges over 50 ps dynamics (same time interval employed in Figures 6-9) for both wild type and mutant molecules. Distances displayed refer to those involving the charged $\mathrm{C}$ and $\mathrm{N}$ terminal ends and the positive charges on lysine and arginine, in all their possible combinations. In Table 1 , it is possible to see that the constrained wild type helical conformations lead to a greater average distance between the terminal oppositely charged ends, as compared to those observed for the random coiled peptide as well as to the conformation after release of the helical 
TABLE 1: Distances $(\AA)$ between Charges in Wild Type and $\Delta 78 r_{1}$ Mutant Peptides

\begin{tabular}{|c|c|c|c|}
\hline & $\mathrm{Arg}^{+}$ & $\mathrm{Lys}^{+}$ & terminal $\mathrm{COO}^{-}$ \\
\hline \multicolumn{4}{|c|}{ Random $\Delta 78 \mathrm{r}_{1}$ (from $t=50$ to $100 \mathrm{ps}$ ) } \\
\hline terminal $\mathrm{NH}_{3}{ }^{+}$ & $9.1 \pm 0.9$ & $9.1 \pm 1.7$ & $10.4 \pm 1.2$ \\
\hline $\mathrm{Arg}^{+}$ & 0.0 & $11.6 \pm 1.5$ & $4.5 \pm 1.8$ \\
\hline Lys $7^{+}$ & & 0.0 & $13.3 \pm 2.1$ \\
\hline \multicolumn{4}{|c|}{ Random WT (from $t=250$ to $300 \mathrm{ps}$ ) } \\
\hline terminal $\mathrm{NH}_{3}{ }^{+}$ & $8.1 \pm 0.8$ & $7.0 \pm 0.8$ & $6.3 \pm 1.0$ \\
\hline $\mathrm{Arg6}^{+}$ & 0.0 & $6.8 \pm 0.5$ & $13.0 \pm 1.1$ \\
\hline Lys $7^{+}$ & & 0.0 & $9.4 \pm 0.8$ \\
\hline \multicolumn{4}{|c|}{$\begin{array}{l}\text { Constrained Zwitterionic Helical } \\
\text { WT (from } t=150 \text { to } 200 \mathrm{ps} \text { ) }\end{array}$} \\
\hline terminal $\mathrm{NH}_{3}{ }^{+}$ & $13.5 \pm 1.4$ & $11.3 \pm 0.9$ & $28.4 \pm 1.5$ \\
\hline $\mathrm{Arg6}^{+}$ & 0.0 & $10.3 \pm 0.8$ & $21.6 \pm 2.9$ \\
\hline${\text { Lys } 7^{+}}^{+}$ & & 0.0 & $27.6 \pm 1.4$ \\
\hline
\end{tabular}

Zwitterionic WT after Release of

\begin{tabular}{lccc}
\multicolumn{5}{c}{ Helical Constraints (from $t=250$ to $300 \mathrm{ps}$ ) } \\
terminal $\mathrm{NH}_{3}{ }^{+}$ & $12.3 \pm 0.8$ & $8.0 \pm 0.7$ & $10.0 \pm 1.1$ \\
Arg6 $^{+}$ & 0.0 & $6.2 \pm 0.5$ & $9.0 \pm 0.6$ \\
Lys7 $^{+}$ & & 0.0 & $6.7 \pm 0.5$ \\
\hline & & & \\
\hline & Arg6 & Lys7 & terminal COOH \\
\hline
\end{tabular}

\begin{tabular}{lccc} 
& Arg6 $^{+}$ & Lys $^{+}$ & terminal COOH \\
\hline \multicolumn{4}{c}{ Constrained Terminal Neutral } \\
& Helical WT (from $t=50$ to 100 ps) \\
terminal NH & & \\
Arg6 $^{+}$ & $10.4 \pm 0.7$ & $14.9 \pm 1.3$ & $16.8 \pm 1.2$ \\
Lys7 $^{+}$ & 0.0 & $9.6 \pm 1.3$ & $18.9 \pm 1.1$ \\
& & 0.0 & $21.4 \pm 2.1$
\end{tabular}

\begin{tabular}{lccc}
\multicolumn{4}{c}{ Terminal Neutral WT after Release of } \\
Helical Constraints (from $t=150$ to $200 \mathrm{ps)}$ \\
terminal NH \\
Arg6 $^{+}$ & $10.9 \pm 0.9$ & $14.6 \pm 1.1$ & $19.3 \pm 0.8$ \\
Lys7 $^{+}$ & 0.0 & $10.5 \pm 0.9$ & $16.0 \pm 0.8$ \\
& & 0.0 & $10.5 \pm 1.5$
\end{tabular}

\begin{tabular}{lccr}
\multicolumn{4}{c}{ Terminal Neutral WT after Release of } \\
Helical Constraints (from $t=250$ to $300 \mathrm{ps)}$ \\
terminal $\mathrm{NH}_{2}$ & $11.2 \pm 0.9$ & $17.1 \pm 1.4$ & $19.2 \pm 2.2$ \\
Arg6 $^{+}$ & 0.0 & $11.8 \pm 1.2$ & $16.5 \pm 1.0$ \\
Lys7 $^{+}$ & & 0.0 & $9.9 \pm 1.2$
\end{tabular}

constraints. The geometrical constraints represent, in fact, a stabilizing factor in terms of maintenance of the helix, since they hinder the attraction between oppositely charged groups, which we consider is the main factor inducing helix disruption in our modeled systems. That can be seen in Table 1 confronting the $28.4 \AA$ average distance (between $\mathrm{C}$ and $\mathrm{N}$ terminal ends) observed for the constrained helix, with $6.3 \AA$ for the random coiled, nonconstrained peptide and $10.0 \AA$ after release of constraints from the originally helical peptide. The random coiled mutant sequence has also shown a comparatively lower value $(10.4 \AA)$ for the $\mathrm{COO}^{-}$to $\mathrm{NH}_{3}{ }^{+}$distance. Distances between the charged Arg and Lys residues and the terminal carbonyl are also drastically reduced in the random coiled and in the nonconstrained helix. The effect of the terminal charges can be better evaluated analyzing the results obtained for a neutral terminal form of the wild type peptide (Table 1). Here, a much less pronounced effect in terms of distances is observed when the helical constraints are removed, even for times as long as 200 ps after the release of the constraints has been applied.

The overall patterns of migration and energy profiles observed throughout this work indicate that the wild type molecule is clearly more inclined to stabilize at the interface proximity, whereas the mutant sequence undergoes a fast kind of interface crossing, with no tendency toward stabilization. Such a behavior is not surprising for a functionally defective molecule. Random coiled conformations have shown a more pronounced affinity for the hydrophobic phase, whereas the helical peptides presented a rather amphiphilic behavior, with a tendency to stabilize at the interface. In addition to that, no proper conditions were found in the modeling that could lead to the

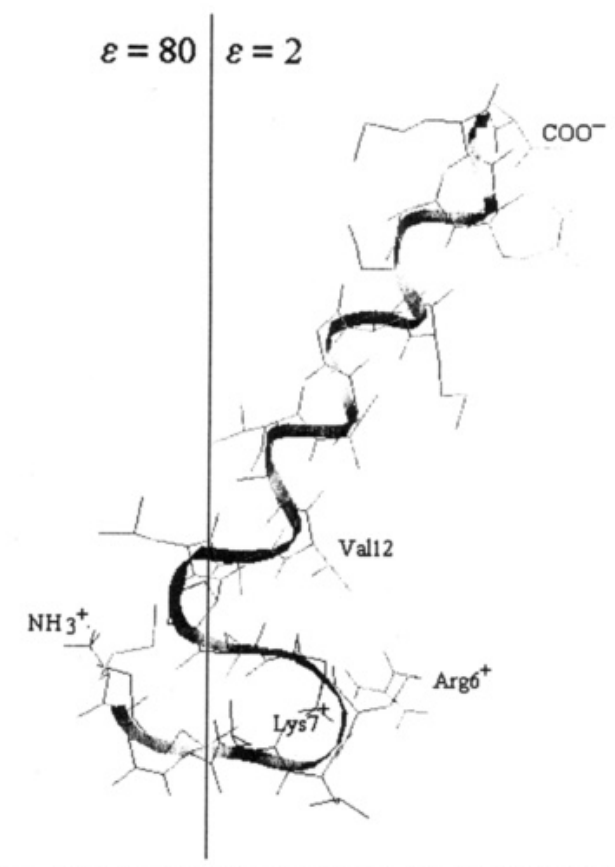

Figure 10. Wild type peptide with constrained helix in zwitterionic form. Coordinates generated at time $=200 \mathrm{ps}$.

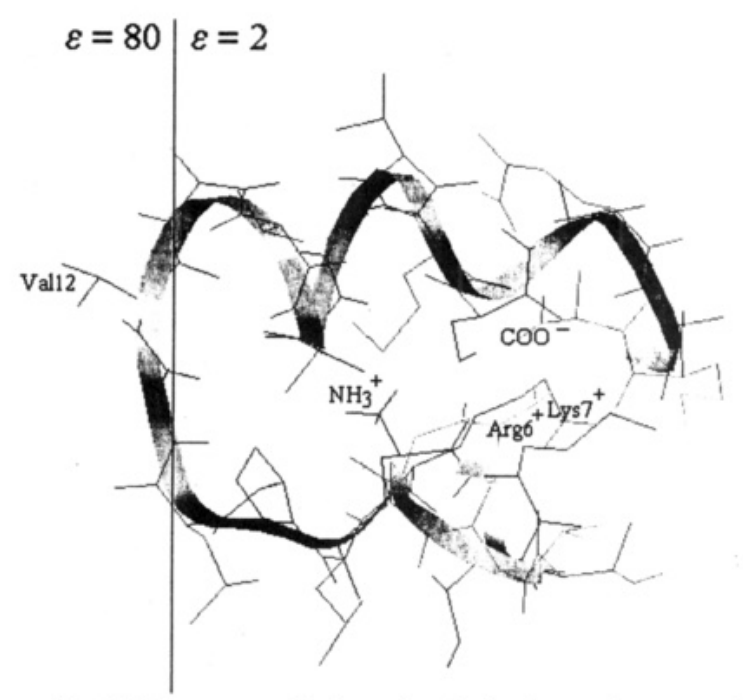

Figure 11. Wild type peptide in zwitterionic form after removal of helical constraints. Coordinates generated at time $=300 \mathrm{ps}$.

helix stabilization for the mutant molecule. That is in accordance with data ${ }^{10}$ referring to lower helix stability in that peptide.

Molecular graphic representation of the zwitterionic wild type peptide in constrained helical conformation, after 200 ps of dynamics in a dielectrically discontinuous medium, is shown in Figure 10. The rather stretched helical axis encompasses around two-thirds of the molecule and is buried in the nonpolar phase. In spite of the constraints, a nonnegligible drift on the helical dihedral angles can be observed around the Pro residue. The charged $\mathrm{N}$ terminal end is in the polar phase, whereas the two other positively charged residues (Lys7 and Arg6) can be found in the interior of the nonpolar phase. That can be related to their tendency to interact with the negatively charged $\mathrm{C}$ terminal group and with the negative end of the helix dipole. The peptide assumes an inclined position (ca. $30^{\circ}$ ) relative to the interface. When constraints are released (Figure 11), the helix stands a considerable disruption, with the positively charged residues approximating further the $\mathrm{C}$ terminal end. The charged $\mathrm{N}$ terminal group can now be found also in the nonpolar 


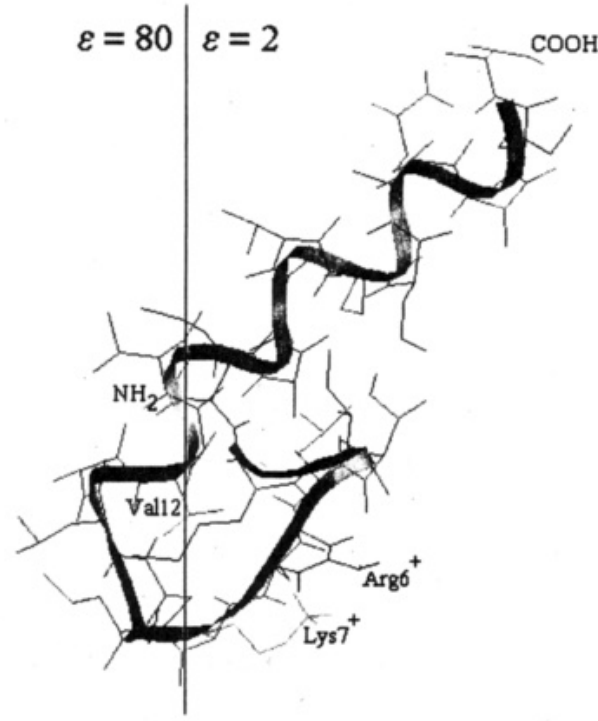

Figure 12. Wild type peptide with constrained helix in neutral terminal form. Coordinates generated at time $=100$ ps.

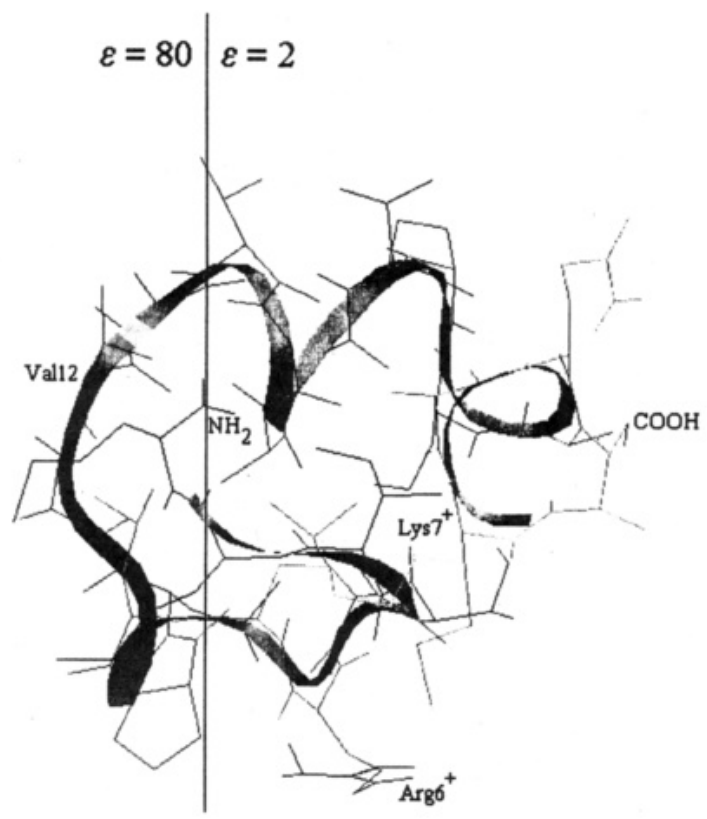

Figure 13. Wild type peptide in neutral terminal form after removal of helical constraints. Coordinates generated at time $=300$ ps.

phase, in a position closer to the negatively charged $\mathrm{C}$ terminal group, and the peptide assumes a ca. $90^{\circ}$ angle relative to the interface. The molecule folds in two, with the valine residue in position 12 showing a shift from its original position in the nonpolar phase. It now sticks into the polar phase.

Figure 12 displays a graphic representation of the wild type peptide in its neutral terminal form, with constrained helix, at $100 \mathrm{ps}$. The general picture is not very different from that observed for the zwitterionic molecule (Figure 10). The terminal neutral peptide is also inclined, now ca. $45^{\circ}$, relative to the interface, with the two charged residues (Lys7 and Arg6) also buried in the nonpolar phase. That may be caused by the presence of the helix dipole (with its negative end toward the C terminal region). Val12 still is at a helical disrupted region and is located in the polar phase. The general peptide folding shows that it is mainly the $\mathrm{C}$ terminal region which bears most of the remaining helical conformation after helical constraints are released (Figure 13). Helix disruption around Val12 is observed, with the molecule assuming a folded conformation, driven by the dipole interaction forces taking place in the nonpolar medium between the positively charged residues and the negative end of the helix dipole. That drift in the valine backbone dihedral angles can be also noticed in the plots presented in Figures 8 and 9.

Figures 10-13 indicate that the wild type peptide stabilizes at the interface, in both its zwitterionic and neutral terminal forms, undergoing a partial helix disruption in the process. The remaining helix is mainly restricted to the $\mathrm{C}$ terminal region, encompassing around 12 residues after the constraints are released. The fact that NMR indicates a 17-residue-long helix for that peptide ${ }^{10}$ does not represent a conflict with our results, since the NMR results were obtained for the peptide in a continuous dielectric medium, namely a 50/50 (in volume) TFE/ $\mathrm{H}_{2} \mathrm{O}$ mixture. The introduction of the dielectric discontinuity in our simulation studies seems to have caused a consistent conformational change in the systems modeled, leading the molecule to a particular kind of folding where a backbone turn is clearly observed. That may not be observed in the absence of the dielectric discontinuity. The main driving force for the occurrence of that turn is the intense electrostatic interaction between oppositely charged regions, made particularly intense in the medium with lower $(\epsilon=2)$ dielectric constant. The positively charged residues and the charged $\mathrm{N}$ terminal in the case of the zwitterionic molecule will interact with the negative region of the helix dipole and with the $\mathrm{C}$ terminal end in the case of the zwitterionic molecule. TFE/ $\mathrm{H}_{2} \mathrm{O}$ mixtures represent a continuous environment, with an intermediate dielectric constant between those used in our discontinuous interfacial model (where $\epsilon=80$ and $\epsilon=2$ were applied), and therefore would not necessarily be expected to induce the particular kind of interfacial charge rearrangements and intense dipolar interactions observed in the dielectrically discontinuous medium that we have used. The dielectric discontinuity is certainly a closer approximation to the membrane environment than a continuous medium, and our modeling results point to the importance of such an aspect being taken into consideration, in the appraisal of conformational features of interfacial peptides.

\section{Conclusions}

The application of the THOR software on signaling peptides has allowed the modeling of a complex macromolecular translational system across a hydrophilic/hydrophobic interface, as well as the study of the effects of some structural features, such as primary sequence, secondary structure, and terminal charges, on patterns of migration and stability features.

Different patterns of behavior have been observed for the wild type and mutant sequences, as would be expected of the behavior of homologous but functionally distinct species. It was shown that the random coiled peptides, both the wild type and the mutant, tend to cross the interface going into the nonpolar phase, although the former shows a small tendency to stay at the interfacial region. In contrast, when a helical conformation is imposed, the wild type tends to remain at the interface. Our results confirm the close relationship between helical conformation and the location of the functional peptide at the membranewater interface, in accordance with literature data ${ }^{10}$ that point to a correlation between helical conformation and peptide functionality and location at the membrane. The interface has been shown to induce particular patterns of peptide conformation and charge distribution across the membrane region. Interesting conformational features were revealed in the presence of the interface, as the occurrence of a backbone turn with consequent partial helix disruption in the wild type peptide, as a result of both the dielectric discontinuity and strong dipolar interactions in the nonpolar medium. 
Although many questions are still open, as for example how the presence of a second membrane/water interface would affect the peptides movements, the present results seem to be useful for an initial understanding of the relationship between conformation and location of peptides in the membrane environment.

Acknowledgment. E.P.G.A. and P.G.P. acknowledge a research and a doctorate fellowship from CNPq and CAPES, respectively. The authors are grateful to Carlos E. M. Hill for the peptide graphic images, to the Centro de Super Computação, UFRJ, Brazil, for access to their computational facilities, and to the Fundação Universitária José Bonifácio, Brazil, for supporting local computational facilities.

\section{References and Notes}

(1) Mundim, K. C.; Pascutti, P. G.; Bisch, P. M. Manuscript in preparation.

(2) Karplus, M.; Petsko, G. Nature 1990, 347, 631.

(3) Katahira, M.; Sugeta, H.; Kyogoku, Y.; Fujii, S. Biochemistry 1990, 29,7214 .

(4) Haran, G.; Haas, E.; Rapaport, D. C. J. Phys. Chem. 1994, 98, 0294.
(5) Clore, G. M.; Gronenborn, A. M.; Brunger, A. T.; Karplus, M. $J$. Mol. Biol. 1985, 186, 435.

(6) Lipkowitz, K. B.; Peterson, M. A. Chem. Rev. 1993, 93, 2463.

(7) Merz, Jr., K. M., Le Grand, S. M., Eds. The Protein Folding Problem and Tertiary Structure Prediction; Birkhäuser: Berlin, 1994.

(8) Perico, A. Biopolymers 1989, 28, 1527.

(9) Ediger, M. D. Annu. Rev. Phys. Chem. 1991, 2, 225.

(10) Bruch, M. D.; Gierasch, L. M. J. Biol. Chem. 1990, 265, 3851.

(11) Jones, J. D.; McKnight, C. J.; Gierasch, L. M. J. Bioenerg. Biomembr. 1990, 22, 213.

(12) Bruch, M. D.; McKnight, C. J.; Gierasch, L. M. Biochemistry 1989. 28,8554 .

(13) Landry, S. J.; Gierasch, L. M. Trends Biochem. Sci. 1991, 16, 159.

(14) van Gunsteren, W. F.; Berendsen, H. J. C. Groningen Molecular Simulation (GROMOS) Library Manual; Biomos: Groningen, 1987.

(15) Berendsen, H. J. C.; van Gusteren, W. F. In Molecular Dynamics Simulation of Statistical-Mechanical Systems; North-Holland: Amsterdam, 1986; pp 43-65.

(16) Allen, M. P.; Tildesley, D. J. Computer Simulation of Liquids; Clarendon Press: Oxford, 1987.

(17) Jackson, J. D. In Classical Electrodynamics, 2nd ed.: John Wiley \& Sons: New York, 1975.

(18) Davis, M. E.; McCammon, J. A. J. Comput. Chem. 1990, 2, 401. (19) Arêas, E. P. G.; Pascutti, P. G.; Schreier, S.; Mundin, K. C.; Bisch, P. M. Braz. J. Med. Biol. Res. 1994, 27, 527.

JP951021W 